Article

\title{
Design, Synthesis, and Cytotoxicity of 5-Fluoro-2-methyl-6-(4-aryl-piperazin-1-yl) Benzoxazoles
}

\author{
Thuraya Al-Harthy ${ }^{1}$, Wajdi Michel Zoghaib ${ }^{1}$, Maren Pflüger ${ }^{2}$, Miriam Schöpel ${ }^{3}$, Kamil Önder ${ }^{4}$, \\ Maria Reitsammer ${ }^{4}$, Harald Hundsberger ${ }^{2}$, Raphael Stoll ${ }^{3}$ and Raid Abdel-Jalil ${ }^{1, *}$ \\ 1 Chemistry Department, College of Science, Sultan Qaboos University, Muscat 123, Oman; \\ thurayalharthi@gmail.com (T.A.-H.); zoghaibw@squ.edu.om (W.M.Z.) \\ 2 IMC Fachhochschule Krems University of Applied Sciences Krems, Piaristengasse 1, Krems A-3500, Austria; \\ maren.pflueger@fh-krems.ac.at (M.P.); harald.hundsberger@fh-krems.ac.at (H.H.) \\ 3 Biomolecular NMR, Ruhr University of Bochum, Bochum 44780, Germany; \\ miriam.schoepel@ruhr-uni-bochum.de (M.S.); raphael.stoll@ruhr-uni-bochum.de (R.S.) \\ 4 Research Program for Rational Drug Design in Dermatology and Rheumatolog, Department of Dermatology, \\ General Hospital of Salzburg, Paracelsus Medical, University of Salzburg, Müllner Hauptstraße 48, \\ Salzburg A-5020, Austria; oender@procomcure.com (K.Ö.); m.reitsammer@salk.at (M.R.) \\ * Correspondence: jalil@squ.edu.om; Tel.: +968-2414-1483
}

Academic Editor: Derek J. McPhee

Received: 5 August 2016; Accepted: 22 September 2016; Published: 27 September 2016

\begin{abstract}
To design new compounds suitable as starting points for anticancer drug development, we have synthesized a novel series of benzoxazoles with pharmaceutically advantageous piperazine and fluorine moieties attached to them. The newly synthesized benzoxazoles and their corresponding precursors were evaluated for cytotoxicity on human A-549 lung carcinoma cells and non-cancer HepaRG hepatocyes. Some of these new benzoxazoles show potential anticancer activity, while two of the intermediates show lung cancer selective properties at low concentrations where healthy cells are unaffected, indicating a selectivity window for anticancer compounds.
\end{abstract}

Keywords: benzoxazole; piperazine; fluorine Cytotoxicity; A-549 lung carcinoma cells; HepaRG hepatocytes

\section{Introduction}

Despite all the progress in biomedical research, cancer remains a leading cause of death worldwide. Therefore, many efforts have been made to find new therapeutic agents that could potentially improve cancer therapy by using more selective and less toxic drugs.

Heterocyclic compounds are considered to be the most interesting drug-based structures in medicinal chemistry due to their widespread presence in lead compounds. Specifically, benzoxazoles have received much attention in recent years because of their broad spectrum of biological activities. Recent studies show that several benzoxazole analogues exhibit a number of biological activities including anticancer [1], antifungal [2], antituberculosis [3], mPGES-1 inhibitor [4], 5-HT receptor antagonist [5], CETP inhibitor [6], and antiplasmodial [7] activities.

Broad therapeutic spectrum compounds intrinsically possess a certain amount of cytotoxicity. Structural modification on lead anticancer compounds may eliminate or reduce cytotoxicity to a minimum level. Usually, a plethora of analogues are synthesized to decipher the underlying structure activity relationship (SAR) and to identify more selective, less toxic, and ADME-improved leads. Often, substitutions found to be bioactively advantageous on a certain active scaffold can be introduced to other scaffolds leading to the enhancement of bioactivity $[8,9]$. Fluorine and piperazine are common appendages and substituents in medicinal chemistry due to their immense utilities in drug design and their unique bioactivities [10,11]. 
The introduction of fluorine and piperazine appendages played a vital role in enhancing the antimicrobial activities of the quinolone drugs, e.g., ciprofloxacin ${ }^{\circledR}$ (1) (Figure 1), the most active antibiotic drug on the market [12]. Similarly, we have successfully synthesized a variety of heterocyclic systems incorporating fluorine and piperazine moieties, such as quinoxaline [13] (2), benzimidazole [14] (3), quinazolinone [15] (4), and recently benzothiazole [16] (5). Some derivatives of our systems showed very promising biological activities, which were attributed to the presence of both appendages. In this study, we combined the benzoxazole scaffold with both piperazine and fluorine moieties and evaluated their potential in anticancer activity.

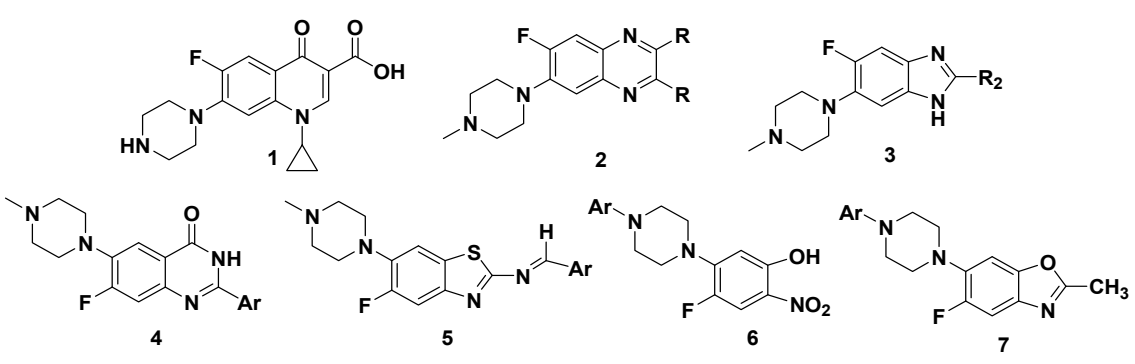

Figure 1. Examples of heterocyclic systems that contain fluorine and piperazine.

Based on that, and as a part of our ongoing research in developing new potentially bioactive heterocyclic compounds, this study describes the synthesis of new 4-fluoro-5-(substituted phenyl-piperazin-1-yl)-2-nitro-phenoles (6) and their corresponding 5-fluoro-6-(substituted phenyl-piperazin-1-yl)-benzoxazoles (7) functionalized with both piperazine and fluorine moieties.

\section{Results and Discussion}

\subsection{Chemistry}

A new series of benzoxazole derivatives was synthesized using a multi-step protocol that involved regioselective nitration and piperazinylation, followed by a one-pot in-situ reductive cyclization step as depicted in Scheme 1.

To start with, the commercially available 3-chloro-4-fluorophenol (8) was nitrated using mild nitration conditions. The choice of a mild nitration step was effective and led to a shorter synthetic procedure compared to the use of strong acidic conditions. In fact, nitration yielded 5-chloro-4-fluoro2-nitrophenol (9) and its 3-chloro-4-fluoro-2,6-dintirophenol (10) counterpart. Carefully controlling the reaction temperature, we were able to isolate 9 in a $60 \%$ yield after purification using flash column chromatography.

Subsequently, the piperazinylation of 9 using excess substituted phenyl $N$-piperazine yielded the key intermediates $\mathbf{6} \mathbf{a}-\mathbf{j}$. Changing the solvent from dimethylsulfoxide to toluene simplified the work and considerably improved the yield up to $83 \%$ for $\mathbf{6} \mathbf{a}, \mathbf{6} \mathbf{b}, \mathbf{6 d}, \mathbf{6 e}, \mathbf{6 g}, \mathbf{6} \mathbf{i}$, and $\mathbf{6} \mathbf{j}$ as shown in Table 1 . For the preparation of $\mathbf{6 c}, \mathbf{6 f}$, and $\mathbf{6} \mathbf{h}$ chlorobenzene was the solvent of choice, due to poor solubility of the corresponding aryl-piperazines in toluene nonetheless using chlorobenzene required a longer reaction time. Purification using column chromatography was required to remove unreacted $N$-piperazines. 
<smiles>Cc1ccc(O)cc1Cl</smiles>

(ii)<smiles>O=[N+]([O-])c1cc(O)c(N2CCN(c3ccccc3)CC2)cc1F</smiles>

6a-j<smiles>[R]c1ccc(N2CCN(c3cc4oc(C)nc4cc3F)CC2)cc1</smiles>

$7 a-j$

Scheme 1. Synthesis of 5-fluoro-6-(substituted phenyl-piperazin-1-yl)-benzoxazoles; reagents and conditions: (i) $15 \% \mathrm{HNO}_{3} / \mathrm{AcOH}, 0{ }^{\circ} \mathrm{C}$, (ii) aryl piperazine, refluxing toluene or chlorobenzene (iii) In $\mathrm{AcOH}$, benzene, trimethyl orthoacetate.

Table 1. Data for the synthesis of benzoxazoles and their precursors.

\begin{tabular}{|c|c|c|c|c|c|c|c|c|}
\hline Compound & $\mathbf{R}$ & Time (h) & Yield *\% & Solvent ** & Compound & Time (h) & Yield *\% & Solvent ** \\
\hline $6 \mathbf{a}$ & $\mathrm{H}$ & 5.5 & 72 & Toluene & $7 a$ & 4 & 71 & Benzene \\
\hline $6 b$ & $p-\mathrm{CH}_{3}$ & 2 & 73 & Toluene & $7 b$ & 3 & 53 & Benzene \\
\hline $6 d$ & $p-\mathrm{F}$ & 2 & 73 & Toluene & $7 d$ & 2.5 & 51 & Benzene \\
\hline $6 e$ & $p-\mathrm{OCH}_{3}$ & 1.5 & 27 & Toluene & $7 e$ & 1 & 70 & Benzene \\
\hline $6 f$ & $o-\mathrm{OH}$ & 3 & 71 & Chlorobenzene & $7 f$ & 1.5 & 55 & Benzene \\
\hline $6 i$ & $m-\mathrm{OCH}_{3}$ & 2 & 73 & Toluene & $7 \mathbf{i}$ & 1 & 77 & Benzene \\
\hline $6 j$ & $3,4-\mathrm{Cl}$ & 5 & 33 & Toluene & $7 \mathbf{j}$ & 1 & 75 & Benzene \\
\hline
\end{tabular}

* The yield was calculated after purification by crystallization or column chromatography. ${ }^{* *}$ Solvent at reflux temperature.

The cyclization of intermediates $(\mathbf{6 a}-\mathbf{j})$ was achieved by an indium-mediated reaction using indium/acetic acid followed by addition of trimethyl orthoaceate to form the corresponding benzoxazole derivatives $(\mathbf{7 a - j})$. This one-pot, reductive cyclization simplified and shortened the synthetic procedure and provided yields ranging from good to very good (53\%-75\%) after recrystallization as shown in Table 1. All the newly synthesized compounds were characterized using spectroscopic techniques. High-resolution mass spectrometry shows the correct molecular ion peaks. The formation of 3-chloro-4-fluoro-2,6-dinitrophenol (10) as a byproduct during the nitration step simultaneously with 9 was confirmed by ${ }^{1} \mathrm{H}-\mathrm{NMR}$ in which one and two types of ${ }^{1} \mathrm{H}$ signals were evident in the spectra of $\mathbf{1 0}$ and $\mathbf{9}$, respectively. In the ${ }^{1} \mathrm{H}-\mathrm{NMR}$ spectra of compounds $(\mathbf{7 a}-\mathbf{j})$, the methyl proton signals appeared as singlets around $\delta 2.54-2.60 \mathrm{ppm}$. The $\mathrm{H}-4$ protons were the 
most deshielded protons, showing a large ortho coupling with the adjacent fluorine $J_{\mathrm{H}-\mathrm{F}}=11.2-12.0 \mathrm{~Hz}$ compared with H-7 meta coupling $J_{\mathrm{H}-\mathrm{F}}=5.0-8.0 \mathrm{~Hz}$. Piperazinyl protons resonated as broad singlets around $\delta 3.10-3.36 \mathrm{ppm}$. The disappearance of the exchangeable signals in intermediates $(\mathbf{6} \mathbf{a}-\mathbf{j})$ around $\delta 10.40-10.80 \mathrm{ppm}$ confirmed the successful cyclization to benzoxazoles. In ${ }^{13} \mathrm{C}-\mathrm{NMR}$ spectra of compounds $(7 \mathbf{a}-\mathbf{j})$, the most deshielded carbon was C-2, resonating around $\delta 163.0-164.6 \mathrm{ppm}$. The methyl carbons appear in the upfield region around $\delta 13.5-14.6 \mathrm{ppm}$ and the piperazine methylene carbons resonated around $\delta 48.0-52.0 \mathrm{ppm}$.

\subsection{Cytotoxicity}

The cytotoxicity of prepared benzoxazoles $(7 \mathbf{a}-\mathbf{j})$ and intermediates $(\mathbf{6} \mathbf{a}-\mathbf{j}$ and $\mathbf{9})$ were measured by applying the CellTiter-Glo ${ }^{\circledR}$ luminescent cell proliferation assay (G755A, Promega, Madison, WI, USA). This method permits the determination of viable cells numbers in the culture based on quantitation of the ATP present, which denotes the presence of metabolically active cells [17]. In vitro cytotoxicity analysis was overall performed following the protocol as described in "Guidance Document on Using in Vitro Data to Estimate in Vivo Starting Doses for Acute Toxicity Based on Recommendations" [18]. Human A-549 cells are human lung cancer epithelial cells, which were chosen as the tumor model, whereas the HepaRG ${ }^{\mathrm{TM}}$ cell line is a classic hepatic cell line that retains many characteristics of primary human hepatocytes. HepaRG ${ }^{\mathrm{TM}}$ cells are terminally differentiated. In order to obtain $\mathrm{CT}_{50}$ values and detect a dose-dependent impact for each of the twelve test compounds, concentrations of 0, 0.1, 1.0, $10,50,100,250$, and $500 \mu \mathrm{M}$ were analyzed with both cell lines, and doxorubicine $\left(\mathrm{IC}_{50}=5.1 \mu \mathrm{M}\right.$ for HepaRG $^{\mathrm{TM}}$ cells) was used as standard.

As shown in Table 2, we observed visible solubility problems for some compounds at concentrations of $50 \mu \mathrm{M}, 100 \mu \mathrm{M}$, and $250 \mu \mathrm{M}$, while $\mathbf{6 d}$ and $7 \mathbf{f}$ were completely soluble at all concentrations. Therefore, a $\mathrm{CT}_{50}$ value of $\sim 50 \mu \mathrm{M}$ could only be obtained for $6 \mathrm{~d}$, which shows equal toxicity to both cell lines, indicating a non-differential behavior.

Since the majority of test compound solutions with concentrations above $50 \mu \mathrm{M}$ precipitated in cell-culture media, we performed cytotoxicity assays for healthy and cancer cells at $50 \mu \mathrm{M}$. For compounds $\mathbf{6 i}, \mathbf{7} \mathbf{a}, \mathbf{7 d}, \mathbf{7 g}, \mathbf{7 h}, \mathbf{7} \mathbf{i}$, and $\mathbf{7 j}$, it was not possible to draw any conclusion because of the precipitation problem.

Compared to healthy control cells, compounds $\mathbf{6 a}, \mathbf{6 b}, \mathbf{6 g}$, and $\mathbf{6 j}$ showed significant increased toxicity in lung cancer A- 549 cells $>30 \%-40 \%$. Interestingly, two compounds were more toxic to healthy cells compared with lung cancer cells $7 \mathbf{b}$ and $7 \mathbf{j}$.

Even at concentrations as low as $10 \mu \mathrm{M}, \mathbf{6 a}$ and $\mathbf{6 g}$ killed $\sim 40 \%$ of lung cancer cells, whereas all compounds were non-toxic to healthy cells. At a 10- $\mu \mathrm{M}$ concentration, nearly none of the tested compounds was toxic to healthy hepatocytes, but at least four compounds $(\mathbf{6 a}, \mathbf{6 b}, \mathbf{6 g}$, and $\mathbf{6 j})$ were highly toxic to lung cancer cells, killing $30 \%-40 \%$ of all cancer cells at this low concentration. Concentrations below $10 \mu \mathrm{M}$ were not toxic at all, indicating a small activity window, which has to be further extended through improving efficacy in order to obtain useful compounds. 
Table 2. Cell viability shown in \% for benzoxazole derivatives and their intermediates.

\begin{tabular}{|c|c|c|c|c|c|c|c|c|c|c|c|c|c|c|c|c|}
\hline \multicolumn{17}{|c|}{ Concentration (C) $\mu \mathrm{M}$} \\
\hline \multicolumn{9}{|c|}{ Liver Cells, Hepatocytes } & \multicolumn{8}{|c|}{ Lung Cancer Cells } \\
\hline Compound & 0 & 0.1 & 1 & 10 & 50 & 100 & 250 & 500 & $\mathbf{0}$ & 0.1 & 1 & 10 & 50 & 100 & 250 & 500 \\
\hline $6 a$ & 100 & 86 & 97 & 112 & 90 & 76 & & & 100 & 97 & 104 & 64 & 61 & 58 & & \\
\hline $6 b$ & 100 & 107 & 123 & 120 & 97 & 96 & & & 100 & 99 & 100 & 68 & 65 & & & \\
\hline $6 c$ & 100 & 95 & 101 & 110 & 101 & 89 & & & 100 & 104 & 103 & 102 & 94 & & & \\
\hline $6 d$ & 100 & 94 & 87 & 101 & 57 & 62 & 44 & 13 & 100 & 103 & 111 & 78 & 58 & 54 & 46 & 34 \\
\hline $6 e$ & 100 & 107 & 110 & 104 & 74 & 79 & & & 100 & 101 & 104 & 96 & 71 & & & \\
\hline $6 f$ & 100 & 105 & 104 & 110 & 76 & & & & 100 & 97 & 100 & 93 & 60 & 46 & & \\
\hline $6 \mathrm{~g}$ & 100 & 110 & 109 & 112 & 87 & & & & 100 & 96 & 96 & 62 & 51 & 42 & & \\
\hline $6 \mathrm{~h}$ & 100 & 117 & 109 & 108 & 83 & 58 & & & 100 & 106 & 106 & 105 & 87 & & & \\
\hline $6 \mathrm{i}$ & 100 & 79 & 95 & 99 & & & & & 100 & 95 & 93 & 96 & 69 & & & \\
\hline $6 \mathbf{j}$ & 100 & 119 & 115 & 81 & 102 & 108 & & & 100 & 98 & 94 & 65 & 62 & & & \\
\hline $7 a$ & 100 & 101 & 97 & 94 & & & & & 100 & 101 & 96 & 90 & 60 & & & \\
\hline $7 b$ & 100 & 105 & 99 & 91 & 63 & 60 & & & 100 & 92 & 94 & 92 & 87 & 75 & & \\
\hline $7 \mathrm{c}$ & 100 & 98 & 107 & 101 & 93 & & & & 100 & 92 & 97 & 101 & 90 & 83 & & \\
\hline $7 d$ & 100 & 91 & 94 & 85 & & & & & 100 & 98 & 98 & 87 & 87 & 82 & & \\
\hline $7 \mathbf{e}$ & 100 & 107 & 108 & 91 & 71 & & & & 100 & 93 & 92 & 75 & 74 & 68 & & \\
\hline $7 f$ & 100 & 106 & 97 & 86 & 59 & 60 & 53 & 54 & 100 & 103 & 99 & 91 & 91 & 95 & 83 & 63 \\
\hline $7 \mathrm{~g}$ & 100 & 96 & 106 & 87 & & & & & 100 & 96 & 92 & 92 & 73 & 63 & & \\
\hline $7 \mathrm{~h}$ & 100 & 100 & 102 & 99 & & & & & 100 & 100 & 93 & 97 & 95 & 90 & & \\
\hline $7 \mathbf{i}$ & 100 & 100 & 102 & 91 & & & & & 100 & 105 & 102 & 88 & 76 & 75 & & \\
\hline $7 \mathbf{j}$ & 100 & 100 & 90 & 85 & 65 & 27 & 7 & 7 & 100 & 98 & 94 & 81 & 70 & 47 & 28 & \\
\hline
\end{tabular}




\section{Materials and Methods}

\subsection{Chemistry}

Reagents were obtained from commercial sources and used without further purification. All solvents were purchased from Sigma Aldrich (Munich, Germany) except for benzene and chlorobenzene, which were purchased from BDH. The staring materials, 3-chloro-4-fluorophenol and trimethyl orthoacetate, were purchased from Alfa Aesar (Munich, Germany).

The newly synthesized compounds were characterized using IR, ${ }^{1} \mathrm{H}-\mathrm{NMR},{ }^{13} \mathrm{C}-\mathrm{NMR}, \mathrm{HRMS}$, and a melting point. IR spectra $\left(4000-400 \mathrm{~cm}^{-1}\right)$ were recorded as $\mathrm{KBr}$ discs on a Perkin-Elmer Spectrum BX FTIR spectrophotometer (Akron, OH, USA). The ${ }^{1} \mathrm{H}-\mathrm{NMR}$ spectra were measured on Varian (Palo Alto, CA, USA) $400 \mathrm{MHz}$, and ${ }^{13} \mathrm{C}$-NMR spectra were recorded at $100 \mathrm{MHz}$ using TMS as an internal standard assigned as zero ppm, and chemical shifts $\delta$ were given in part per million (ppm) downfield from TMS. The EI and FAB high-resolution mass spectra were recorded on a Finnigan MAT 312 mass spectrometer (ThermoFisher Scientific, Waltham, MA, USA) Melting points were determined on an SMP 10 Stuart Scientific apparatus (Keison Products, Essex, UK). The reactions were monitored by thin layer chromatography (TLC) using silica gel plates, and the components were visualized by UV light. Flash column chromatography was carried out using silica gel 60 (70-230 mesh) (Aldrich, Munich, Germany) as the stationary phase.

5-chloro-4-fluoro-2-nitrophenol (9) and 3-chloro-4-fluoro-2,6-dintirophenol (10): for the nitrating solution, $15 \% \mathrm{HNO}_{3} / \mathrm{AcOH}$ was prepared and kept at $0{ }^{\circ} \mathrm{C}$. 3-chloro-4-fluorophenol (8) $(5.0 \mathrm{~g}, 34 \mathrm{mmol})$ was added in small portions to the solution and stirred for $1 \mathrm{~h}$ at $0{ }^{\circ} \mathrm{C}$. The reaction mixture poured into an ice-water bath, filtered by suction, washed thoroughly with water, and then dried and purified by column chromatography $10 \% \mathrm{MeOH} / \mathrm{CH}_{2} \mathrm{Cl}_{2}$.

5-Chloro-4-fluoro-2-nitro-phenol (9) (yield 60\%). m.p.: 82-83 ${ }^{\circ} \mathrm{C}$. FTIR (KBr): 3471, 1629, 1530, 1330, 1247, $1150 \mathrm{~cm}^{-1} .{ }^{1} \mathrm{H}-\mathrm{NMR}\left(400 \mathrm{MHz}, \mathrm{CDCl}_{3}\right) \delta(\mathrm{ppm}): 10.40$ (bs, 1H, O-H), 7.91 (d, J = 8.4 Hz, H-6), 7.26 (d, $J=12.0 \mathrm{~Hz}, \mathrm{H}-3) .{ }^{13} \mathrm{C}-\mathrm{NMR}\left(100 \mathrm{MHz}, \mathrm{CDCl}_{3}\right) \delta$ (ppm): 152.2(C), $151.7(\mathrm{C}), 150.0(\mathrm{C}), 132.5(\mathrm{C}), 121.8$ (CH), $112.1(\mathrm{CH})$. MS (ESI) [M] $m / z$ calcd for $\mathrm{C}_{6} \mathrm{H}_{3} \mathrm{ClFNO}_{3}$ : 190.98; found: 189.75 .

3-Chloro-4-fluoro-2,6-dintirophenol (10) (yield 20\%). ${ }^{1} \mathrm{H}-\mathrm{NMR}\left(400 \mathrm{MHz}, \mathrm{CDCl}_{3}\right) \delta$ (ppm): 10.60 (bs, $1 \mathrm{H}$, $\mathrm{O}-\mathrm{H}), 8.11$ (d, $J=9.2 \mathrm{~Hz}, \mathrm{H}-3$ ). MS (ESI) [M] $m / z$ calcd for $\mathrm{C}_{6} \mathrm{H}_{2} \mathrm{ClFN}_{2} \mathrm{O}_{5}$ : 235.96; found: 234.79 .

3.1.1. General Procedure for Synthesis of 4-Fluoro-5-(substitutedphenyl-piperazin-1-yl)-2-nitro-phenol $(6 a, 6 b, 6 d, 6 e, 6 g, 6 i, 6 j)$

5-Chloro-4-fluoro-2-nitro-phenol (9) $(0.500 \mathrm{~g}, 2.61 \mathrm{mmol})$ was dissolved in toluene $(5 \mathrm{~mL})$, and the corresponding aryl piperazine $(13.1 \mathrm{mmol})$ was then added and refluxed for $2-6 \mathrm{~h}$. Afterwards, water was added and extracted with dichloromethane $(3 \times 30 \mathrm{~mL})$, and the organic layer was concentrated, dried over sodium sulfate, and purified by flash column chromatography.

4-Fluoro-2-nitro-5-(4-phenyl-piperazin-1-yl)-phenol (6a) (yield 72\%, 40\% hexane $/ \mathrm{CH}_{2} \mathrm{Cl}_{2}$ ). m.p.: 197-198 ${ }^{\circ} \mathrm{C}$. FTIR (KBr): 3377, 1634, 1508, 1388, $1225 \mathrm{~cm}^{-1} .{ }^{1} \mathrm{H}-\mathrm{NMR}\left(400 \mathrm{MHz}, \mathrm{CDCl}_{3}\right) \delta$ (ppm): 10.90 (bs, $1 \mathrm{H}$, O-H), 7.74 (d, $J=13.2 \mathrm{~Hz}, 1 \mathrm{H}, \mathrm{H}-3), 6.46$ (d, $J=7.6 \mathrm{~Hz}, 1 \mathrm{H}, \mathrm{H}-6), 3.52$ (s, 2H, piperazine), 3.34 (s, 2H, piperazine). ${ }^{13} \mathrm{C}-\mathrm{NMR}\left(100 \mathrm{MHz}, \mathrm{CDCl}_{3}\right) \delta(\mathrm{ppm}): 153.9(\mathrm{C}), 150.6(\mathrm{C}), 148.3(\mathrm{C}), 145.9(\mathrm{C}), 129.3$ $(\mathrm{CH}), 120.7(\mathrm{C}), 116.5(\mathrm{CH}), 112.0(\mathrm{CH}), 111.8(\mathrm{CH}), 105.2(\mathrm{CH}), 49.31\left(\mathrm{CH}_{2}\right), 49.26\left(\mathrm{CH}_{2}\right)$. HRMS (EI) $m / z$ calcd for $\mathrm{C}_{16} \mathrm{H}_{16} \mathrm{FN}_{3} \mathrm{O}_{3}$ : 317.1176; found: 317.1163 .

4-Fluoro-2-nitro-5-(4-p-tolyl-piperazin-1-yl)-phenol (6b) (yield 73\%, $\mathrm{CH}_{2} \mathrm{Cl}_{2}$ ). m.p.: $178-179{ }^{\circ} \mathrm{C}$. FTIR (KBr): 3354, 1631, 1511, 1390, $1220 \mathrm{~cm}^{-1} .{ }^{1} \mathrm{H}-\mathrm{NMR}\left(400 \mathrm{MHz}, \mathrm{CDCl}_{3}\right) \delta$ (ppm): 10.84 (bs, 1H, O-H), 7.68 $(\mathrm{d}, J=13.2 \mathrm{~Hz}, 1 \mathrm{H}, \mathrm{H}-3), 6.83(\mathrm{~d}, J=7.2 \mathrm{~Hz}, 1 \mathrm{H}, \mathrm{H}-6), 3.46$ (s, 2H, piperazine), 3.22 (s, 2H, piperazine), 2.23 (s, 1H, $\left.\mathrm{CH}_{3}-\mathrm{Ph}\right) .{ }^{13} \mathrm{C}-\mathrm{NMR}\left(100 \mathrm{MHz} \mathrm{CDCl}_{3}\right) \delta(\mathrm{ppm}): 154.0(\mathrm{C}), 148.4(\mathrm{C}), 148.3(\mathrm{C}), 145.9(\mathrm{C})$, $129.9(\mathrm{CH}), 124.8(\mathrm{C}), 124.7(\mathrm{C}), 117.0(\mathrm{CH}), 112.0(\mathrm{CH}), 105.3(\mathrm{CH}), 49.8\left(\mathrm{CH}_{2}\right), 49.2\left(\mathrm{CH}_{2}\right), 20.5\left(\mathrm{CH}_{3}\right)$. HRMS (ESI) $\left[\mathrm{M}+\mathrm{H}^{+}\right] \mathrm{m} / \mathrm{z}$ calcd for $\mathrm{C}_{17} \mathrm{H}_{18} \mathrm{FN}_{3} \mathrm{O}_{3}$ : 332.1405; found: 332.1407. 
4-Fluoro-5-[4-(4-fluoro-phenyl)-piperazin-1-yl]-2-nitro-phenol (6d) (yield $73 \%, \mathrm{CH}_{2} \mathrm{Cl}_{2}$ ). m.p.: $151-152{ }^{\circ} \mathrm{C}$. FTIR (KBr): 3309, 3103, 1637, 1535, $1244 \mathrm{~cm}^{-1} .{ }^{1} \mathrm{H}-\mathrm{NMR}\left(400 \mathrm{MHz}, \mathrm{CDCl}_{3}\right) \delta(\mathrm{ppm}): 10.88(\mathrm{bs}, 1 \mathrm{H}$, O-H), $7.74(\mathrm{~d}, J=13.6 \mathrm{~Hz}, 1 \mathrm{H}, \mathrm{H}-3), 6.47(\mathrm{~d}, J=7.60 \mathrm{~Hz}, 1 \mathrm{H}, \mathrm{H}-6), 3.51(\mathrm{~s}, 2 \mathrm{H}$, piperazine), $3.25(\mathrm{~s}, 2 \mathrm{H}$, piperazine). ${ }^{13} \mathrm{C}-\mathrm{NMR}(100 \mathrm{MHz} \mathrm{CDCl} 3) \delta$ (ppm): 158.9 (C), 156.5 (C), 153.9 (C), $148.3(\mathrm{C}), 146.0(\mathrm{C})$, $124.9(\mathrm{C}), 118.5(\mathrm{CH}), 115.7(\mathrm{CH}), 111.8(\mathrm{CH}), 105.3(\mathrm{CH}), 50.2\left(\mathrm{CH}_{2}\right), 49.3\left(\mathrm{CH}_{2}\right)$. HRMS (EI) $\mathrm{m} / z$ calcd for $\mathrm{C}_{16} \mathrm{H}_{15} \mathrm{~F}_{2} \mathrm{~N}_{3} \mathrm{O}_{3}$ : 335.1081; found: 335.1066 .

4-Fluoro-5-[4-(4-methoxy-phenyl)-piperazin-1-yl]-2-nitro-phenol (6e) (yield 27\%, $\mathrm{CH}_{2} \mathrm{Cl}_{2}$ ). m.p.: 202-204 ${ }^{\circ} \mathrm{C}$. FTIR (KBr): 3448, 2933, 1638, 1515, $1225 \mathrm{~cm}^{-1} .{ }^{1} \mathrm{H}-\mathrm{NMR}\left(400 \mathrm{MHz}, \mathrm{CDCl}_{3}\right) \delta$ (ppm): 10.84 (bs, $1 \mathrm{H}$, $\mathrm{O}-\mathrm{H}), 7.68(\mathrm{~d}, J=13.2 \mathrm{~Hz}, 1 \mathrm{H}, \mathrm{H}-3), 6.41(\mathrm{~d}, J=7.6 \mathrm{~Hz}, 1 \mathrm{H}, \mathrm{H}-6), 3.72\left(\mathrm{~s}, 1 \mathrm{H}, \mathrm{OCH}_{3}\right), 3.47(\mathrm{~s}, 2 \mathrm{H}$, piperazine), $3.17\left(\mathrm{~s}, 2 \mathrm{H}\right.$, piperazine). ${ }^{13} \mathrm{C}-\mathrm{NMR}\left(100 \mathrm{MHz}, \mathrm{CDCl}_{3}\right) \delta(\mathrm{ppm}): 153.9(\mathrm{C}), 148.3(\mathrm{C}), 145.9$ (C), $122.5(\mathrm{C}), 121.4(\mathrm{C}), 119.2(\mathrm{C}), 114.7(\mathrm{CH}), 112.0(\mathrm{CH}), 111.8(\mathrm{CH}), 105.5(\mathrm{CH}), 55.6\left(\mathrm{CH}_{3}\right), 51.4$ $\left(\mathrm{CH}_{2}\right), 49.0\left(\mathrm{CH}_{2}\right)$. HRMS (EI) $\mathrm{m} / z$ calcd for $\mathrm{C}_{17} \mathrm{H}_{18} \mathrm{FN}_{3} \mathrm{O}_{4}$ : 347.1281; found: 347.1264 .

4-Fluoro-5-[4-(2-fluoro-phenyl)-piperazin-1-yl]-2-nitro-phenol (6g) (yield $83 \%, \mathrm{CH}_{2} \mathrm{Cl}_{2}$ ). m.p.: $180-181{ }^{\circ} \mathrm{C}$. FTIR (KBr): 3457, 2928, 1636, 1508, $1265 \mathrm{~cm}^{-1} .{ }^{1} \mathrm{H}-\mathrm{NMR}\left(400 \mathrm{MHz}, \mathrm{CDCl}_{3}\right) \delta(\mathrm{ppm}): 10.82(\mathrm{bs}, 1 \mathrm{H}$, O-H), 7.69 (d, J = 13.2 Hz, 1H, H-3), $6.42(\mathrm{~d}, J=7.6 \mathrm{~Hz}, 1 \mathrm{H}, \mathrm{H}-6), 3.51$ (s, 2H, piperazine), 3.25 (s, 2H, piperazine). ${ }^{13} \mathrm{C}-\mathrm{NMR}\left(100 \mathrm{MHz}, \mathrm{CDCl}_{3}\right) \delta$ (ppm): 157.0 (C), 154.5 (C), 154.0 (C), 148.5 (C), 145.9 (C), 139.3 (C), $124.6(\mathrm{CH}), 123.3(\mathrm{CH}), 119.1(\mathrm{CH}), 116.2(\mathrm{CH}), 111.8(\mathrm{CH}), 105.4(\mathrm{CH}), 50.2\left(\mathrm{CH}_{2}\right), 49.5\left(\mathrm{CH}_{2}\right)$. HRMS (EI) $\mathrm{m} / \mathrm{z}$ calcd for $\mathrm{C}_{16} \mathrm{H}_{15} \mathrm{~F}_{2} \mathrm{~N}_{3} \mathrm{O}_{3}$ : 335.1081; found: 335.1070 .

4-Fluoro-5-[4-(3-methoxy-phenyl)-piperazin-1-yl]-2-nitro-phenol (6i) (yield $73 \%, \mathrm{CH}_{2} \mathrm{Cl}_{2}$ ). m.p.: $148-149{ }^{\circ} \mathrm{C}$. FTIR (KBr): 3426, 2937, 1629, 1504, $1209 \mathrm{~cm}^{-1} .{ }^{1} \mathrm{H}-\mathrm{NMR}\left(400 \mathrm{MHz}, \mathrm{CDCl}_{3}\right) \delta$ (ppm): 10.83 (bs, $1 \mathrm{H}$, $\mathrm{O}-\mathrm{H}), 7.68(\mathrm{~d}, J=13.6 \mathrm{~Hz}, 1 \mathrm{H}, \mathrm{H}-3), 6.52(\mathrm{~d}, J=8.0 \mathrm{~Hz}, 1 \mathrm{H}, \mathrm{H}-6), 3.74\left(\mathrm{~s}, 1 \mathrm{H}, \mathrm{OCH}_{3}\right), 3.45(\mathrm{~s}, 2 \mathrm{H}$, piperazine), 3.28 (s, 2H, piperazine). ${ }^{13} \mathrm{C}-\mathrm{NMR}\left(100 \mathrm{MHz}, \mathrm{CDCl}_{3}\right) \delta(\mathrm{ppm}): 160.6(\mathrm{C}), 153.9$ (C), 151.9 (C), $148.3(\mathrm{C}), 145.9(\mathrm{C}), 130.0(\mathrm{CH}), 124.8(\mathrm{C}), 112.0(\mathrm{CH}), 111.8(\mathrm{CH}), 109.1(\mathrm{CH}), 105.2(\mathrm{CH}), 103.0(\mathrm{CH})$, $55.2\left(\mathrm{CH}_{3}\right), 49.2\left(\mathrm{CH}_{2}\right), 49.1\left(\mathrm{CH}_{2}\right)$. HRMS (EI) $\mathrm{m} / z$ calcd for $\mathrm{C}_{17} \mathrm{H}_{18} \mathrm{FN}_{3} \mathrm{O}_{4}$ : 347.1281; found: 347.1297.

5-[4-(3,4-Dichloro-phenyl)-piperazin-1-yl]-4-fluoro-2-nitro-phenol (6j) (yield 33\%, $\mathrm{CH}_{2} \mathrm{Cl}_{2}$ ). m.p.: 211-213 ${ }^{\circ} \mathrm{C}$. FTIR (KBr): 3448, 2924, 1634, 1513, $1207 \mathrm{~cm}^{-1} .{ }^{1} \mathrm{H}-\mathrm{NMR}\left(400 \mathrm{MHz}, \mathrm{CDCl}_{3}\right) \delta$ (ppm): 10.80 (bs, 1H, O-H), $7.70(\mathrm{~d}, J=13.6 \mathrm{~Hz}, 1 \mathrm{H}, \mathrm{H}-3), 6.42$ (d, $J=7.6 \mathrm{~Hz}, 1 \mathrm{H}, \mathrm{H}-6), 3.49$ (s, 2H, piperazine), 3.30 (s, 2H, piperazine). ${ }^{13} \mathrm{C}-\mathrm{NMR}\left(100 \mathrm{MHz}, \mathrm{CDCl}_{3}\right) \delta$ (ppm): 153.9 (C), 149.8 (C), $148.3(\mathrm{C}), 148.0(\mathrm{C}), 145.9$ (C), $133.0(\mathrm{C}), 130.7(\mathrm{CH}), 120.1(\mathrm{C}), 117.9(\mathrm{CH}), 115.9(\mathrm{CH}), 112.1(\mathrm{CH}), 105.4(\mathrm{CH}), 49.0\left(\mathrm{CH}_{2}\right), 48.8$ $\left(\mathrm{CH}_{2}\right)$. HRMS (EI) $\mathrm{m} / z$ calcd for $\mathrm{C}_{16} \mathrm{H}_{14} \mathrm{C}_{12} \mathrm{FN}_{3} \mathrm{O}_{3}$ : 385.0396; found: 385.0374 .

3.1.2. General Procedure for Synthesis 5-[4-( $o / m / p$-Hydroxy-phenyl)-piperazin-1-yl]-4-fluoro2-nitro-phenol (6c, 6f, 6h)

5-Chloro-4-fluoro-2-nitro-phenol (9) $(0.250 \mathrm{~g}, 1.31 \mathrm{mmol})$ was dissolved in chlorobenzene $(5 \mathrm{~mL})$, substituted phenyl-piperazine $(6.53 \mathrm{mmol})$ was added and refluxed overnight. The reaction mixture was extracted with water and dichloromethane, and the organic layer was concentrated and purified by flash column chromatography.

4-Fluoro-5-[4-(4-hydroxy-phenyl)-piperazin-1-yl]-2-nitro-phenol (6c) (yield 37\%, 10\% EtOAc/ $\mathrm{CH}_{2} \mathrm{Cl}_{2}$ ). m.p.: $218-219{ }^{\circ} \mathrm{C}$. FTIR (KBr): 3475, 3117, 1631, 1515, 1242, $\mathrm{cm}^{-1} .{ }^{1} \mathrm{H}-\mathrm{NMR}\left(400 \mathrm{MHz}, \mathrm{CDCl}_{3}\right.$ with 2 drops of $\left.\mathrm{CD}_{3} \mathrm{OD}\right) \delta(\mathrm{ppm}): 10.81$ (bs, $\left.1 \mathrm{H}, \mathrm{O}-\mathrm{H}\right), 7.67$ (d, $\left.J=13.2 \mathrm{~Hz}, 1 \mathrm{H}, \mathrm{H}-3\right), 6.42(\mathrm{~d}, J=7.6 \mathrm{~Hz}$, $1 \mathrm{H}, \mathrm{H}-6), 3.46$ (s, 2H, piperazine), 3.13 (s, $2 \mathrm{H}$, piperazine). ${ }^{13} \mathrm{C}-\mathrm{NMR}\left(100 \mathrm{MHz}, \mathrm{CDCl}_{3}\right.$ with 2 drops of $\left.\mathrm{CD}_{3} \mathrm{OD}\right) \delta(\mathrm{ppm}): 152.8(\mathrm{C}), 147.5(\mathrm{C}), 147.4(\mathrm{C}), 147.3(\mathrm{C}), 144.9(\mathrm{C}), 123.8(\mathrm{C}), 118.5(\mathrm{CH}), 115.0$ $(\mathrm{CH}), 110.7(\mathrm{CH}), 104.4(\mathrm{CH}), 50.4\left(\mathrm{CH}_{2}\right), 50.4\left(\mathrm{CH}_{2}\right)$. HRMS (EI) $\mathrm{m} / z$ calcd for $\mathrm{C}_{16} \mathrm{H}_{16} \mathrm{FN}_{3} \mathrm{O}_{4}$ : 333.1125; found: 331.1141.

4-Fluoro-5-[4-(2-hydroxy-phenyl)-piperazin-1-yl]-2-nitro-phenol (6f) (yield 71\%, $\mathrm{CH}_{2} \mathrm{Cl}_{2}$ ). m.p.: $176-178{ }^{\circ} \mathrm{C}$. FTIR (KBr): 3452, 2923, 1636, 1520, $1227 \mathrm{~cm}^{-1} .{ }^{1} \mathrm{H}-\mathrm{NMR}\left(400 \mathrm{MHz}, \mathrm{CDCl}_{3}\right) \delta$ (ppm): 10.81 (bs, $1 \mathrm{H}$, $\mathrm{O}-\mathrm{H}), 7.70(\mathrm{~d}, J=13.2 \mathrm{~Hz}, 1 \mathrm{H}, \mathrm{H}-3), 6.43(\mathrm{~d}, J=8.0 \mathrm{~Hz}, 1 \mathrm{H}, \mathrm{H}-6), 3.45(\mathrm{~s}, 2 \mathrm{H}$, piperazine $), 2.99(\mathrm{~s}, 2 \mathrm{H}$, piperazine). ${ }^{13} \mathrm{C}-\mathrm{NMR}(100 \mathrm{MHz}, \mathrm{DMSO}) \delta$ (ppm): 152.9 (C), 150.6 (C), 147.5 (C), 145.2 (C), 139.7 (C), 
$126.0(\mathrm{C}), 123.7(\mathrm{CH}), 116.1(\mathrm{CH}), 119.9(\mathrm{CH}), 119.3(\mathrm{CH}), 112.6(\mathrm{CH}), 106.5(\mathrm{CH}), 50.3\left(\mathrm{CH}_{2}\right), 49.7\left(\mathrm{CH}_{2}\right)$. HRMS (EI) $m / z$ calcd for $\mathrm{C}_{16} \mathrm{H}_{16} \mathrm{FN}_{3} \mathrm{O}_{4}$ : 333.1125; found: 333.1111 .

4-Fluoro-5-[4-(3-hydroxy-phenyl)-piperazin-1-yl]-2-nitro-phenol (6h) (yield 28\%, 25\% EtOAc/ $\mathrm{CH}_{2} \mathrm{Cl}_{2}$ ). m.p.: $207-208{ }^{\circ} \mathrm{C}$. FTIR (KBr): 3448, 2924, 1636, 1506, $1260 \mathrm{~cm}^{-1} .{ }^{1} \mathrm{H}-\mathrm{NMR}\left(400 \mathrm{MHz}, \mathrm{CDCl}_{3}\right) \delta$ (ppm): 10.83 (bs, 1H, O-H), 7.69 (d, J = 13.2 Hz, 1H, H-3), 7.11 (m, 1H, H-6), 3.46 (s, 2H, piperazine), 3.28 (s, 2H, piperazine). ${ }^{13} \mathrm{C}-\mathrm{NMR}(100 \mathrm{MHz}, \mathrm{DMSO}) \delta$ (ppm): 158.5 (C), 152.7 (C), 147.5 (C), 147.3 (C), 145.1 (C), $130.1(\mathrm{CH}), 126.1(\mathrm{C}), 112.9(\mathrm{CH}), 107.2(\mathrm{CH}), 107.0(\mathrm{CH}), 106.4(\mathrm{CH}), 103.0(\mathrm{CH}), 49.4\left(\mathrm{CH}_{2}\right), 48.4$ $\left(\mathrm{CH}_{2}\right)$. HRMS (EI) $m / z$ calcd for $\mathrm{C}_{16} \mathrm{H}_{16} \mathrm{FN}_{3} \mathrm{O}_{4}$ : 333.1125; found: 333.1131 .

3.1.3. General Procedure for Synthesis of 5-Fluoro-6-(substitutedphenyl-piperazin-1-yl)benzoxazoles $(7 \mathbf{a}-\mathbf{j})$

Fluoro-5-(substituted phenyl-piperazin-1-yl)-2-nitro-phenol $(\mathbf{6 a}-\mathbf{j})(1 \mathrm{mmol})$ was added to a mixture of indium powder $(8 \mathrm{mmol})$ and acetic acid $(20 \mathrm{mmol})$ in benzene $(2 \mathrm{~mL})$. Trimethyl orthoacetate $(8 \mathrm{mmol})$ in benzene $(2 \mathrm{~mL})$ was then added to the reaction mixture, stirred, and refluxed for $1-4 \mathrm{~h}$. The reaction mixture was cooled to room temperature, washed with saturated sodium bicarbonate, extracted with dichloromethane $(3 \times 10 \mathrm{~mL})$, and washed with water followed by saturated sodium chloride solution (brine). The organic extracts were dried over sodium sulfate anhydrous and concentrated using a rotary evaporator. The residue was then recrystallized from absolute ethanol to yield the corresponding benzoxazole.

5-Fluoro-2-methyl-6-(4-phenylpiperazin-1-yl)-benzo[d]oxazole (7a) (yield 71\%). m.p.: 153-154 ${ }^{\circ} \mathrm{C}$. FTIR (KBr): 2848, 1638, $1242 \mathrm{~cm}^{-1} .{ }^{1} \mathrm{H}-\mathrm{NMR}\left(400 \mathrm{MHz}, \mathrm{CDCl}_{3}\right) \delta(\mathrm{ppm}): 7.27(\mathrm{~m}, 1 \mathrm{H}, \mathrm{H}-4), 7.06(\mathrm{~d}, J=6.8 \mathrm{~Hz}$, $1 \mathrm{H}, \mathrm{H}-7), 3.35$ (s, 2H, piperazine), $3.22\left(\mathrm{~s}, 2 \mathrm{H}\right.$, piperazine), $2.54\left(\mathrm{~s}, 3 \mathrm{H}, \mathrm{CH}_{3}\right) .{ }^{13} \mathrm{C}-\mathrm{NMR}(100 \mathrm{MHz}$, $\left.\mathrm{CDCl}_{3}\right) \delta(\mathrm{ppm}): 163.1(\mathrm{C}), 153.9(\mathrm{C}), 151.5(\mathrm{C}), 150.0(\mathrm{C}), 137.2(\mathrm{C}), 134.9(\mathrm{C}), 128.2(\mathrm{CH}), 119.3(\mathrm{CH})$, $115.4(\mathrm{CH}), 105.4(\mathrm{CH}), 99.5(\mathrm{CH}), 50.2\left(\mathrm{CH}_{2}\right), 48.6\left(\mathrm{CH}_{2}\right), 13.5\left(\mathrm{CH}_{3}\right)$. HRMS (EI) $\mathrm{m} / z$ calcd for $\mathrm{C}_{18} \mathrm{H}_{18} \mathrm{FN}_{3} \mathrm{O}$ : 311.1434; found: 311.1423 .

5-Fluoro-2-methyl-6-(4-p-tolyl-piperazin-1-yl)-benzooxazole (7b) (yield 53\%). m.p.: 168-170 ${ }^{\circ} \mathrm{C}$. FTIR (KBr) 1636, $1265 \mathrm{~cm}^{-1} .{ }^{1} \mathrm{H}-\mathrm{NMR}\left(400 \mathrm{MHz}, \mathrm{CDCl}_{3}\right) \delta(\mathrm{ppm}): 7.27$ (d, $\left.J=11.6 \mathrm{~Hz}, 1 \mathrm{H}, \mathrm{H}-4\right), 7.06(\mathrm{~m}, 1 \mathrm{H}, \mathrm{H}-7)$, $3.28\left(\mathrm{~s}, 2 \mathrm{H}\right.$, piperazine), 3.19 (s, 2H, piperazine), $2.54\left(\mathrm{~s}, 3 \mathrm{H}, \mathrm{CH}_{3}\right), 2.23\left(\mathrm{~s}, 3 \mathrm{H}, \mathrm{CH}_{3}-\mathrm{Ph}\right) .{ }^{13} \mathrm{C}-\mathrm{NMR}$ $\left(100 \mathrm{MHz}, \mathrm{CDCl}_{3}\right) \delta$ (ppm): 164.1 (C), 152.5 (C), 149.0 (C), 147.5 (C), 138.2 (C), 135.9 (C), 135.8 (C), 129.7 $(\mathrm{CH}), 116.8(\mathrm{CH}), 106.2(\mathrm{CH}), 100.5(\mathrm{CH}), 51.2\left(\mathrm{CH}_{2}\right)$, 50.1( $\left(\mathrm{CH}_{2}\right), 20.5\left(\mathrm{CH}_{3}\right), 14.6\left(\mathrm{CH}_{3}\right)$. HRMS (EI) $m / z$ calcd for $\mathrm{C}_{19} \mathrm{H}_{20} \mathrm{FN}_{3} \mathrm{O}$ : 325.1590; found: 325.1577 .

4-[4-(5-Fluoro-2-methyl-benzooxazol-6-yl)-piperazin-1-yl]-phenol (7c) (yield 63\%). m.p.: 188-191 ${ }^{\circ} \mathrm{C}$. FTIR (KBr): 3466, 2937, 1640, $1267 \mathrm{~cm}^{-1} .{ }^{1} \mathrm{H}-\mathrm{NMR}(400 \mathrm{MHz}, \mathrm{DMSO}) \delta$ (ppm): 8.90 (bs, 1H, O-H), 7.48 (d, $J=12.0 \mathrm{~Hz}, 1 \mathrm{H}, \mathrm{H}-4), 7.38(\mathrm{~d}, J=7.2 \mathrm{~Hz}, 1 \mathrm{H}, \mathrm{H}-7), 3.36$ (s, 2H, piperazine), 3.10 (s, 2H, piperazine), 2.54 (s, 3H, $\left.\mathrm{CH}_{3}\right) .{ }^{13} \mathrm{C}-\mathrm{NMR}(100 \mathrm{MHz}, \mathrm{DMSO}) \delta(\mathrm{ppm}): 164.6(\mathrm{C}), 154.6(\mathrm{C}), 152.2(\mathrm{C}), 147.6(\mathrm{C}), 144.5(\mathrm{C})$, $138.4(\mathrm{C}), 135.5(\mathrm{C}), 118.5(\mathrm{CH}), 115.9(\mathrm{CH}), 106.5(\mathrm{CH}), 101.6(\mathrm{CH}), 51.3\left(\mathrm{CH}_{2}\right), 50.6\left(\mathrm{CH}_{2}\right), 14.6\left(\mathrm{CH}_{3}\right)$. HRMS (EI) $m / z$ calcd for $\mathrm{C}_{18} \mathrm{H}_{18} \mathrm{FN}_{3} \mathrm{O}_{2}$ : 327.1383; found: 327.1391.

5-Fluoro-6-[4-(4-fluoro-phenyl)-piperazin-1-yl]-2-methyl-benzooxazole (7d) (yield 51\%). m.p.: 148-150 ${ }^{\circ} \mathrm{C}$. FTIR (KBr): 2928, 1640, $1267 \mathrm{~cm}^{-1} .{ }^{1} \mathrm{H}-\mathrm{NMR}\left(400 \mathrm{MHz}, \mathrm{CDCl}_{3}\right) \delta(\mathrm{ppm}): 7.27(\mathrm{~d}, J=11.6 \mathrm{~Hz}, 1 \mathrm{H}$, $\mathrm{H}-4), 7.06$ (d, J = 7.2 Hz, 1H, H-7), 3.20 (s, 2H, piperazine), 2.25 (s, 2H, piperazine), $2.54\left(\mathrm{~s}, 3 \mathrm{H}, \mathrm{CH}_{3}\right.$ ). ${ }^{13} \mathrm{C}-\mathrm{NMR}\left(100 \mathrm{MHz}, \mathrm{CDCl}_{3}\right) \delta(\mathrm{ppm}): 164.3(\mathrm{C}), 154.9$ (C), 152.2 (C), 147.6 (C), 144.5 (C), $138.4(\mathrm{C}), 135.5$ (C), $118.5(\mathrm{CH}), 115.9(\mathrm{CH}), 106.5(\mathrm{CH}), 101.6(\mathrm{CH}), 51.6\left(\mathrm{CH}_{2}\right), 51.0\left(\mathrm{CH}_{2}\right), 14.6\left(\mathrm{CH}_{3}\right)$. HRMS (EI) $\mathrm{m} / z$ calcd for $\mathrm{C}_{18} \mathrm{H}_{17} \mathrm{~F}_{2} \mathrm{~N}_{3} \mathrm{O}$ : 329.1340; found: 329.1352 .

5-Fluoro-6-[4-(4-methoxy-phenyl)-piperazin-1-yl]-2-methyl-benzooxazole (7e): (yield 70\%). m.p.: $156-157^{\circ} \mathrm{C}$. FTIR (KBr): 2946, 1636, $1236 \mathrm{~cm}^{-1} .{ }^{1} \mathrm{H}-\mathrm{NMR}\left(400 \mathrm{MHz}, \mathrm{CDCl}_{3}\right) \delta$ (ppm): $7.27(\mathrm{~d}, J=11.6 \mathrm{~Hz}, 1 \mathrm{H}, \mathrm{H}-4)$, $7.06(\mathrm{~d}, J=7.2 \mathrm{~Hz}, 1 \mathrm{H}, \mathrm{H}-7), 3.72\left(\mathrm{~s}, 3 \mathrm{H}, \mathrm{OCH}_{3}\right), 3.21$ (s, 2H, piperazine), 3.20 (s, 2H, piperazine), 2.54 (s, 3H, $\left.\mathrm{CH}_{3}\right) .{ }^{13} \mathrm{C}-\mathrm{NMR}\left(100 \mathrm{MHz}, \mathrm{CDCl}_{3}\right) \delta(\mathrm{ppm}): 164.3$ (C), 153.9 (C), 151.5 (C), 146.5 (C), 144.2 (C), 
137.2 (C), $134.8(\mathrm{C}), 117.6(\mathrm{CH}), 113.5(\mathrm{CH}), 105.2(\mathrm{CH}), 99.5(\mathrm{CH}), 54.5\left(\mathrm{CH}_{3}\right), 50.2\left(\mathrm{CH}_{2}\right), 50.0\left(\mathrm{CH}_{2}\right)$, 13.5 $\left(\mathrm{CH}_{3}\right)$. HRMS (EI) $\mathrm{m} / z$ calcd for $\mathrm{C}_{19} \mathrm{H}_{20} \mathrm{FN}_{3} \mathrm{O}_{2}$ : 341.1540; found: 341.1548 .

2-[4-(5-Fluoro-2-methyl-benzooxazol-6-yl)-piperazin-1-yl]-phenol (7f) (yield 55\%). m.p.: $172-173{ }^{\circ} \mathrm{C}$. FTIR (KBr): 3453, 2928, 1636, $1265 \mathrm{~cm}^{-1} .{ }^{1} \mathrm{H}-\mathrm{NMR}(400 \mathrm{MHz}, \mathrm{CDCl} 3) \delta(\mathrm{ppm}): 7.28$ (d, $J=11.2 \mathrm{~Hz}, 1 \mathrm{H}$, $\mathrm{H}-4), 6.94(\mathrm{~d}, J=5.0 \mathrm{~Hz}, 1 \mathrm{H}, \mathrm{H}-7), 3.24\left(\mathrm{~s}, 2 \mathrm{H}\right.$, piperazine), $3.10\left(\mathrm{~s}, 2 \mathrm{H}\right.$, piperazine), $2.55\left(\mathrm{~s}, 3 \mathrm{H}, \mathrm{CH}_{3}\right)$. ${ }^{13} \mathrm{C}-\mathrm{NMR}\left(100 \mathrm{MHz}, \mathrm{CDCl}_{3}\right) \delta(\mathrm{ppm}): 163.2(\mathrm{C}), 153.9$ (C), 151.5 (C), 150.4 (C), 146.5 (C), $137.0(\mathrm{C}), 134.9$ (C), $125.7(\mathrm{CH}), 120.5(\mathrm{CH}), 119.2(\mathrm{CH}), 113.2(\mathrm{CH}), 105.5(\mathrm{CH}), 99.6(\mathrm{CH}), 51.6\left(\mathrm{CH}_{2}\right), 50.8\left(\mathrm{CH}_{2}\right), 13.6$ $\left(\mathrm{CH}_{3}\right)$. HRMS (EI) $\mathrm{m} / z$ calcd for $\mathrm{C}_{18} \mathrm{H}_{18} \mathrm{FN}_{3} \mathrm{O}_{2}$ : 327.1383; found: 327.1369 .

5-Fluoro-6-(4-(2-fluorophenyl)-piperazin-1-yl)-2-methylbenzo[d]oxazole (7g) (yield 74\%). m.p.: 157-158 ${ }^{\circ} \mathrm{C}$. FTIR (KBr): 2834, 1634, $1271 \mathrm{~cm}^{-1} .{ }^{1} \mathrm{H}-\mathrm{NMR}$ (400 MHz, DMSO) $\delta$ (ppm): 7.49 (d, $J=12.0 \mathrm{~Hz}, 1 \mathrm{H}$, $\mathrm{H}-4), 7.42(\mathrm{~d}, J=7.6 \mathrm{~Hz}, 1 \mathrm{H}, \mathrm{H}-7), 3.36\left(\mathrm{~s}, 2 \mathrm{H}\right.$, piperazine), 3.15 (s, $2 \mathrm{H}$, piperazine), $2.54\left(\mathrm{~s}, 3 \mathrm{H}, \mathrm{CH}_{3}\right)$. ${ }^{13} \mathrm{C}-\mathrm{NMR}(100 \mathrm{MHz}, \mathrm{DMSO}) \delta$ (ppm): 164.7 (C), 156.7 (C), 154.4 (C), 147.6 (C), 140.1 (C), 138.4 (C), 135.7 (C), $125.4(\mathrm{CH}), 123.0(\mathrm{CH}), 119.8(\mathrm{CH}), 116.4(\mathrm{CH}), 106.4(\mathrm{CH}), 101.8(\mathrm{CH}), 51.3\left(\mathrm{CH}_{2}\right), 50.6\left(\mathrm{CH}_{2}\right), 14.6$ $\left(\mathrm{CH}_{3}\right)$. HRMS (EI) $m / z$ calcd for $\mathrm{C}_{18} \mathrm{H}_{17} \mathrm{~F}_{2} \mathrm{~N}_{3} \mathrm{O}$ : 329.1340; found: 329.1353 .

3-[4-(5-Fluoro-2-methyl-benzooxazol-6-yl)-piperazin-1-yl]-phenol (7h) (yield 74\%). m.p.: 211-213 ${ }^{\circ} \mathrm{C}$. FTIR (KBr): 3448, 2941, 1622, $1278 \mathrm{~cm}^{-1} .{ }^{1} \mathrm{H}-\mathrm{NMR}\left(400 \mathrm{MHz}, \mathrm{CDCl}_{3}\right) \delta(\mathrm{ppm}): 7.28(\mathrm{~d}, J=11.6 \mathrm{~Hz}, 1 \mathrm{H}$, $\mathrm{H}-4), 7.12(\mathrm{~d}, J=8.0 \mathrm{~Hz}, 1 \mathrm{H}, \mathrm{H}-7), 3.35\left(\mathrm{~s}, 2 \mathrm{H}\right.$, piperazine), $3.22\left(\mathrm{~s}, 2 \mathrm{H}\right.$, piperazine), $2.55\left(\mathrm{~s}, 3 \mathrm{H}, \mathrm{CH}_{3}\right)$. ${ }^{13} \mathrm{C}-\mathrm{NMR}(100 \mathrm{MHz}, \mathrm{DMSO}) \delta(\mathrm{ppm}): 164.7$ (C), 158.5 (C), 154.6 (C), 152.7 (C), 147.6 (C), $138.4(\mathrm{C})$, 135.6 (C), $130.1(\mathrm{CH}), 107.2(\mathrm{CH}), 106.9(\mathrm{CH}), 106.2(\mathrm{CH}), 103.0(\mathrm{CH}), 101.8(\mathrm{CH}), 51.1\left(\mathrm{CH}_{2}\right), 48.9\left(\mathrm{CH}_{2}\right)$, 14.5 $\left(\mathrm{CH}_{3}\right)$. HRMS (EI) $m / z$ calcd for $\mathrm{C}_{18} \mathrm{H}_{18} \mathrm{FN}_{3} \mathrm{O}_{2}$ : 327.1383; found: 327.1368.

5-Fluoro-6-[4-(3-methoxy-phenyl)-piperazin-1-yl]-2-methyl-benzooxazole (7i) (yield 77\%). m.p.: 124-125 ${ }^{\circ} \mathrm{C}$. FTIR (KBr): 2942, 1636, $1280 \mathrm{~cm}^{-1} .{ }^{1} \mathrm{H}-\mathrm{NMR}\left(400 \mathrm{MHz}, \mathrm{CDCl}_{3}\right) \delta(\mathrm{ppm}): 7.33(\mathrm{~d}, J=11.2 \mathrm{~Hz}, 1 \mathrm{H}, \mathrm{H}-4)$, $7.11(\mathrm{~d}, J=7.2 \mathrm{~Hz}, 1 \mathrm{H}, \mathrm{H}-7), 3.80\left(\mathrm{~s}, 3 \mathrm{H}, \mathrm{OCH}_{3}\right), 3.38(\mathrm{~s}, 2 \mathrm{H}$, piperazine), 3.24 (s, 2H, piperazine), 2.60 $\left(\mathrm{s}, 3 \mathrm{H}, \mathrm{CH}_{3}\right) .{ }^{13} \mathrm{C}-\mathrm{NMR}\left(100 \mathrm{MHz}, \mathrm{CDCl}_{3}\right) \delta(\mathrm{ppm}): 164.2(\mathrm{C}), 160.6(\mathrm{C}), 154.9(\mathrm{C}), 152.5(\mathrm{C}), 147.5(\mathrm{C})$, 138.2 (C), $135.8(\mathrm{C}), 129.9(\mathrm{CH}), 109.1(\mathrm{CH}), 106.2(\mathrm{CH}), 104.8(\mathrm{CH}), 102.8(\mathrm{CH}), 100.5(\mathrm{CH}), 55.2\left(\mathrm{CH}_{3}\right)$, 51.2 $\left(\mathrm{CH}_{2}\right), 49.4\left(\mathrm{CH}_{2}\right), 14.5\left(\mathrm{CH}_{3}\right)$. HRMS (EI) $m / z$ calcd for $\mathrm{C}_{19} \mathrm{H}_{20} \mathrm{FN}_{3} \mathrm{O}_{2}$ : 341.1540; found: 341.1528.

6-[4-(3,4-Dichloro-phenyl)-piperazin-1-yl]-5-fluoro-2-methyl-benzooxazole (7j) (yield 75\%). m.p.: 147-148 ${ }^{\circ} \mathrm{C}$. FTIR (KBr): 2928, 1638, $1272 \mathrm{~cm}^{-1} .{ }^{1} \mathrm{H}-\mathrm{NMR}\left(400 \mathrm{MHz}, \mathrm{CDCl}_{3}\right) \delta$ (ppm): $7.28(\mathrm{~d}, J=11.6 \mathrm{~Hz}, 1 \mathrm{H}$, $\mathrm{H}-4), 7.06(\mathrm{~d}, J=7.2 \mathrm{~Hz}, 1 \mathrm{H}, \mathrm{H}-7), 3.31$ (s, $2 \mathrm{H}$, piperazine), $3.17\left(\mathrm{~s}, 2 \mathrm{H}\right.$, piperazine), $2.55\left(\mathrm{~s}, 3 \mathrm{H}, \mathrm{CH}_{3}\right)$. ${ }^{13} \mathrm{C}-\mathrm{NMR}\left(100 \mathrm{MHz}, \mathrm{CDCl}_{3}\right.$ ) $\delta$ (ppm): 163.3 (C), 153.9 (C), 151.5 (C), 149.3 (C), 146.5 (C), 136.7 (C), 135.2 (C), $131.9(\mathrm{C}), 129.5(\mathrm{CH}), 116.7(\mathrm{CH}), 114.7(\mathrm{CH}), 105.3(\mathrm{CH}), 99.6(\mathrm{CH}), 49.8\left(\mathrm{CH}_{2}\right), 48.1\left(\mathrm{CH}_{2}\right), 13.6$ $\left(\mathrm{CH}_{3}\right)$. HRMS (ESI) $\left[\mathrm{M}+\mathrm{H}^{+}\right] \mathrm{m} / z$ calcd for $\mathrm{C}_{18} \mathrm{H}_{17} \mathrm{C}_{12} \mathrm{FN}_{3} \mathrm{O}$ : 380.0727; found: 380.0729 .

\subsection{Cytotoxicity}

Human cell cultures: Hepatocytes (HepaRG ${ }^{\mathrm{TM}}$ cells) were obtained from Life Technologies, and were cultivated in William's E Medium (Gibco, Life Technologies, Waltham, MA, USA) and supplemented with 1x GlutaMAX ${ }^{\mathrm{TM}}$ plus $1 \times$ HepaRG $^{\mathrm{TM}}$ General Purpose Medium Supplement in $25-\mathrm{cm}^{2}$ or $75-\mathrm{cm}^{2}$ flasks (TPP, Trasadingen, Switzerland) at $37^{\circ} \mathrm{C}$ and $5 \% \mathrm{CO}_{2}$. Lung cancer epithelial cells A-549 were propagated in Epilife Medium (Gibco, Life Technologies; MEPICF) and combined with $\mathrm{CaCl}_{2}$ and 1x-growth-supplement containing penicillin and streptomycin (Gibco, Life Technologies). For sub-culturing, cells were split at a ratio of 1:4 to 1:8. For cryopreservation, cells were frozen in 10\% DMSO in liquid nitrogen.

Human cytotoxicity assay: Human hepatocytes or human lung carcinoma cells were seeded into sterile black clear-bottomed polystyrene Corning ${ }^{\circledR}$ CellBind $^{\circledR}$ 96-well plates (Munich, Germany) at a density of 20,000 cells / well after calculating cell numbers from trypsinized samples in a classical Neubauer Improved hemocytometer (VWR, Munich, Germany). The cells were grown for $24 \mathrm{~h}$ at $37^{\circ} \mathrm{C}$ in a $5 \% \mathrm{CO}_{2}$ atmosphere in Epilife medium (keratinocytes) or William's E medium (hepatocytes). After a 24-h recovery period, media were removed from the wells by aspiration. The cells were then 
immediately exposed to the respective test compounds or reference antibiotics (prepared in advance in $100 \mu \mathrm{L}$ of the same media) for $24 \mathrm{~h} 37^{\circ} \mathrm{C}$ with $5 \% \mathrm{CO}_{2}$. The concentration tested ranged from $0.1 \mu \mathrm{M}$ to $500 \mu \mathrm{M}(0.1 \mu \mathrm{M}, 1 \mu \mathrm{M}, 10 \mu \mathrm{M}, 50 \mu \mathrm{M}, 100 \mu \mathrm{M}, 250 \mu \mathrm{M}$, and $500 \mu \mathrm{M})$ and was formulated as a $100 \times$ concentrated stock each, resulting in a final constant DMSO concentration of $1 \%$ per $100-\mu \mathrm{L}$ sample volume. Test series were each performed in triplicate, and additionally included a vehicle reference (DMSO only) plus another internal control for evaluating overall assay performance. Additionally, cells were visually examined under a light microscope for any morphological abnormalities relative to the untreated cells. Subsequently, cell viability, as an indicator of potential cytotoxicity, was measured by applying the CellTiter-Glo ${ }^{\circledR}$ luminescent cell proliferation assay (G755A, Promega, Madison, WI, USA). This method permits the determination of the number of viable cells in culture based on quantitation of the ATP present, which denotes the presence of metabolically active cells. To this end, $100 \mu \mathrm{L}$ (equal to the volume of culture medium) of the kit-provided CellTiter-Glo ${ }^{\circledR}$ reagent (reconstituted by transferring the thawed CellTiter-Glo ${ }^{\circledR}$ buffer to the lyophilized enzyme and substrate mixture) was directly added to each well with a repetitive dispensing pipette. The contents were then mixed for 2 min at room temperature on an orbital minishaker (IKA ${ }^{\circledR}$ MS3 digital, Staufen, Germany) at $450 \mathrm{rpm}$ to induce efficient cell lysis, and then further incubated for $10 \mathrm{~min}$ at room temperature for stabilization of the resultant luminescent signal. Luminescence was recorded using a compatible multimode reader (GloMax ${ }^{\circledR}$ Multi Detection Platform, Promega Corporation, Madison, WI, USA) with its embedded, preset CellTiter-Glo ${ }^{\circledR}$ parameters (integration time of $0.5 \mathrm{~s}$ ). Output values were expressed as percent cell viability compared with the vehicle (DMSO) control, and $\mathrm{EC}_{50}$ values were calculated from the dose-response curves via nonlinear regression (version 7, Graphpad Prism Software Inc., La Jolla, CA, USA).

\section{Conclusions}

A novel series of 5-fluoro-2-methyl-6-(4-arylpiperazin-1-yl)benzoxazoles (7a-j) incorporating both fluorine and piperazine were prepared in good yields. Initial cytotoxicity analysis of some of the intermediates-4-fluoro-5-(substituted phenylpiperazin-1-yl)-2-nitrophenols (6a-j) - showed promising activities and cell type-dependent cytotoxicity. Although the best activity achieved is still too low, the lung cancer sensitive pattern is a crucial property underlining a therapeutically interesting window, which can be further investigated. The poor solubility of some benzoxazoles could be improved by replacing the aryl-piperazine with $N$-methylpiperazine at position 6 and replacing the methyl moiety at position 2 with a carbamate functional group. Manipulating the benzoxazole structure is now under intensive investigation in our laboratory, and the results will be published in due course. Finally, at least two compounds $(\mathbf{6 d}$ and $\mathbf{6 g}$ ) can be used as starting points for the development of selective anti-cancer compounds in the future.

Acknowledgments: Financial support from Sultan Qaboos University, Oman, is gratefully acknowledged.

Author Contributions: Raid Abdel-Jalil and Wajdi Michel Zoghaib conceived and designed the experiments; Thuraya Al-Harthy performed the experiments and characterization of new compounds; Raphael Stoll's group contributed to the NMR measurements and characterization of the compounds. Kamil Önder, Harald Hundsberger, Maren Pflüger and Maria Reitsammer performed the Cytotoxicity measurements and the discussion of biological data. All authors read and approved the final manuscript.

Conflicts of Interest: The authors declare no conflict of interest.

\section{References}

1. Chanda, K.; Maiti, B.; Yellol, G.S.; Chien, M.-H.; Kuoc, M.-L.; Sun, C.-M. Polymer supported synthesis of novel benzoxazole linked benzimidazoles under microwave conditions: In vitro evaluation of VEGFR-3 kinase inhibition activity. Org. Biomol. Chem. 2011, 9, 1917-1926. [CrossRef] [PubMed]

2. Sheng, C.; Che, X.; Wang, W.; Wang, S.; Cao, Y.; Yao, J.; Miao, Z.; Zhang, W. Design and synthesis of antifungal benzoheterocyclic derivatives by scaffold hopping. Eur. J. Med. Chem. 2011, 46, 1706-1712. [CrossRef] [PubMed] 
3. Klimešováa, V.; Kočía, J.; Waissera, K.; Kaustováb, J.; Möllmannc, U. Preparation and in vitro evaluation of benzylsulfanyl benzoxazole derivatives as potential antituberculosis agents. Eur. J. Med. Chem. 2009, 44, 2286-2293. [CrossRef] [PubMed]

4. $\quad$ Arhancet, G.B.; Walker, D.P.; Metz, S.; Fobian, Y.M.; Heasley, S.E.; Carter, J.S.; Springer, J.R.; Jones, D.E.; Hayes, M.J.; Shaffer, A.F.; et al. Discovery and SAR of PF-4693627, a potent, selective and orally bioavailable mPGES-1 inhibitor for the potential treatment of inflammation. Bioorg. Med. Chem. Lett. 2013, 23, 1114-1119. [CrossRef] [PubMed]

5. Liu, K.G.; Lo, J.R.; Comery, T.A.; Zhang, G.M.; Zhang, J.Y.; Kowal, D.M.; Smith, D.L.; Di, L.; Kerns, E.H.; Schechter, L.E.; et al. Identification of a series of benzoxazoles as potent 5-HT6 ligands. Bioorg. Med. Chem. Lett. 2009, 19, 1115-1117. [CrossRef] [PubMed]

6. Kallashi, F.; Kim, D.; Kowalchick, J.; Park, Y.J.; Hunt, J.A.; Ali, A.; Smith, C.J.; Hammond, M.L.; Pivnichny, J.V.; Tong, X.; et al. 2-Arylbenzoxazoles as CETP inhibitors: Raising HDL-C in cynoCETP transgenic mice. Bioorg. Med. Chem. Lett. 2011, 21, 558-561. [CrossRef] [PubMed]

7. Ongarora, D.S.B.; Gut, J.; Rosenthal, P.J.; Masimirembwa, C.M.; Chibale, K. Benzoheterocyclic amodiaquine analogues with potent antiplasmodial activity: Synthesis and pharmacological evaluation. Bioorg. Med. Chem. Lett. 2012, 22, 5046-5050. [CrossRef] [PubMed]

8. Murty, M.S.R.; Rao, B.R.; Katiki, M.R.; Nath, L.R.; Anto, R.J. Synthesis of piperazinyl benzothiazole/benzoxazole derivatives coupled with 1,3,4-oxadiazole-2-thiol: Novel hybrid heterocycles as anticancer agents. Med. Chem. Res. 2013, 22, 4980-4991. [CrossRef]

9. Shao, K.-P.; Zhang, X.-Y.; Chen, P.-J.; Xue, D.-Q.; He, P.; Ma, L.-Y.; Zheng, J.-X.; Zhang, Q.-R.; Liu, H.-M. Synthesis and biological evaluation of novel pyrimidine-benzimidazol hybrids as potential anticancer agents. Bioorg. Med. Chem. Lett. 2014, 24, 3877-3881. [CrossRef] [PubMed]

10. Shaquiquzzaman, M.; Verma, G.; Marella, A.; Akhter, M.; Akhtar, W.; Khan, M.F.; Tasneem, S.; Alam, M.M. Piperazine scaffold: A remarkable tool in generation of diverse pharmacological agents. Eur. J. Org. Chem. 2015, 102, 487-529. [CrossRef] [PubMed]

11. Filler, R.; Saha, R. Fluorine in medicinal chemistry: a century of progress and a 60-year retrospective of selected highlights. Future Med. Chem. 2009, 1, 777-791. [CrossRef] [PubMed]

12. Sanchez, J.P.; Domagala, J.M.; Hagen, S.E.; Heifetz, C.L.; Hutt, M.P.; Nichols, J.B.; Trehan, A.K. Quinolone Antibacterial Agents. Synthesis and Structure-Activity Relationships of 8-Substituted Quinoline-3-carboxylic Acids and 1,8-Naphthyridine-3-carboxylic Acids. J. Med. Chem. 1988, 31, 983-991. [CrossRef] [PubMed]

13. Abdel-jalil, R.J.; Al-Qawasemeh, R.A.; Voleter, W.; Heeg, P.; El-Abadelah, M.M.; Sabri, S.S. Synthesis and properties of some 2,3-disubstituted 6-fluoro-7-(4-methyl-1-piperazinyl)quinoxalines. J. Heterocycl. Chem. 2009, 37, 1273-1275. [CrossRef]

14. Abu-Elteen, K.H.; Abdel-Jalil, R.J.; Hamad, M.A.; Ghaleb, M.; Khan, K.M.; Voelter, W. fungicidal effects of some derivatives of 2-ferrocenyl-benzimidazoles: A possible template for antifungal drug design. J. Med. Sci. 2008, 8, 673-681. [CrossRef]

15. Abdel-Jalil, R.J.; Aldoqum, H.M.; Ayoub, M.T.; Voelter, W. Synthesis and antitumor activity of 2-aryl-6-fluoro-6-(4-methyl-1-piperazinyl)-4-(3H)-Quinazolinones. Heterocycles 2005, 65, 2061-2070. [CrossRef]

16. Al-Harthy, T.; Abdel-Jalil, R.; Zoghaib, W.; Pflüger, M.; Hofmann, E.; Hundsberger, H. Design and Synthesis of Benzothiazole Schiff Bases of Potential Antitumor Activitiy. Heterocycles 2016, 92, 1282-1292.

17. Crouch, S.; Kozlowski, R.; Slater, K.; Fletcher, J. The use of ATP bioluminescence as a measure of cell proliferation and cytotoxicity. J. Immunol. Methods. 1993, 160, 81-88. [CrossRef]

18. Guidance Document on Using in Vitro Data to Estimate in Vivo Starting Doses for Acute Toxicity. Available online: http://www.epa.gov/hpv/pubs/general/nih2001b.pdf (accessed on 5 November 2014).

Sample Availability: Samples of the compounds are available from the authors.

(C) 2016 by the authors; licensee MDPI, Basel, Switzerland. This article is an open access article distributed under the terms and conditions of the Creative Commons Attribution (CC-BY) license (http://creativecommons.org/licenses/by/4.0/). 